\title{
Extension of Penning trap mass measurements to very short-lived nuclides
}

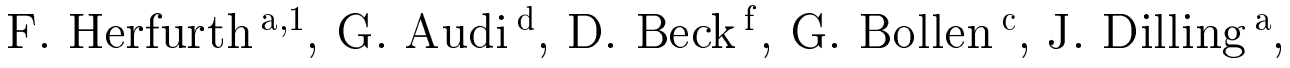 \\ S. Henry ${ }^{\mathrm{d}}$, A. Kellerbauer ${ }^{\mathrm{b}}$, H.-J. Kluge ${ }^{\mathrm{a}}$, V. Kolhinen ${ }^{\mathrm{g}}$, \\ D. Lunney ${ }^{\mathrm{d}}$, R.B. Moore ${ }^{\mathrm{e}}$, C. Scheidenberger ${ }^{\mathrm{a}}$, S. Schwarz ${ }^{\mathrm{b}}$, \\ G. Sikler ${ }^{a}$, J. Szerypo ${ }^{g}$ and the ISOLDE collaboration ${ }^{b}$ \\ ${ }^{a}$ GSI, Planckstraße 1, D-64291 Darmstadt, Germany \\ ${ }^{\mathrm{b}}$ CERN, CH-1211 Geneva 23, Switzerland \\ ${ }^{c}$ Sekt. Physik, Ludwig-Maximilians-Universität München, D-85748 Garching \\ ${ }^{\mathrm{d}}$ CSNSM-IN2P3-CNRS, F-91405 Orsay-Campus, France \\ ${ }^{\mathrm{e}}$ Department of Physics, McGill University, Montréal (Québec) H3A 2T8, Canada \\ ${ }^{\mathrm{f}}$ Instituut voor Kern- en Stralingsfysica, Celestijnenlaan 200 D, B-3001 Leuven, \\ Belgium \\ ${ }^{\mathrm{g}}$ Department of Physics, University of Jyväskylä, PB 35 (Y5), FIN-40351 \\ Jyväskylä, Finland \\ PACS number: 21.10.Dr, 27.30.+t, 32.10.Bi, 07.75.+h
}

\begin{abstract}
Mass measurements on ${ }^{33,34,42,43} \mathrm{Ar}$ have been performed at the ISOLTRAP spectrometer. An accuracy of $\delta m \approx 4 \mathrm{keV}$ has been achieved for all measured isotopes. With ${ }^{33} \mathrm{Ar}$ it is the first time that a nuclide with a half-life shorter than one second has been investigated using a Penning trap. This became possible due to the recently installed linear radio-frequency ion trap system and an improved, faster measurement cycle.
\end{abstract}

Key words: ATOMIC MASSES. Ion guide. Ion trap. Ion cooling. On-line mass spectrometry. Radioactive ion beams. ${ }^{33} \mathrm{Ar}$

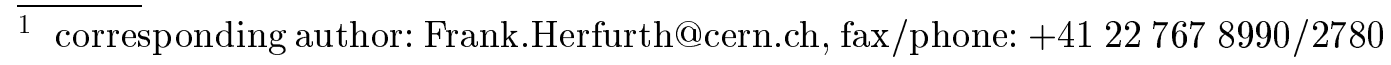

Preprint submitted to Elsevier Preprint

25 April 2001 


\section{Introduction}

Mass measurements of short-lived nuclides have been of great interest for many years. This is because the atomic mass is a gross property of a nuclide that embodies all the effects of the forces that are interplaying within its nucleus. Generally, a large scale survey of the mass surface is needed in order to detect trends in the nuclear binding energy as well as to fit the free parameters of nuclear models. However, in particular, there are nuclides of special interest, spread all over the nuclear chart, that often have very short half-lives. The mass of such nuclides is needed to an extraordinarily high precision for studies such as fundamental tests.

One prominent example is the mass of ${ }^{32} \mathrm{Ar}$. This mass plays an important role for the result of the recently performed $\beta$-neutrino correlation experiment using the $\beta$-delayed proton emission of ${ }^{32} \mathrm{Ar}$ [1]. The significance of the limit set by this experiment on scalar contributions to the weak interaction critically depends on the accuracy of the ${ }^{32}$ Ar mass.

Generally, precision and a very short half-live are contradictory. However, using the ISOLTRAP facility, where a Penning trap is used to perform mass measurements, it has already be shown that for half-lives greater than one second the accuracy does not necessarily depend on the half-live of the measured nuclide. The present work demonstrates that the Penning trap technique is also applicable to nuclides with a half-life well below one second. Indeed, it has been proven that, even for half-lives as short as a few hundred milliseconds, it is possible to obtain an accuracy of $\delta m / m \approx 1 \cdot 10^{-7}$.

\section{Experimental setup}

Figure 1 shows an overview of the layout of the ISOLTRAP mass spectrometer, installed at the online mass separator ISOLDE/CERN [2]. The ISOLTRAP spectrometer consists of a linear radio-frequency ion trap and two Penning traps. The continuous beam delivered from ISOLDE is accumulated, cooled and bunched in the radio-frequency quadrupole (RFQ) ion beam buncher $[3,4]$. The main task of this device is to transform the $60 \mathrm{keV}$ continuous ISOLDE beam into low energy $(2-3 \mathrm{keV})$ low emittance $(\leq 10 \pi \mathrm{mm} \mathrm{mrad})$ ion bunches that can be efficiently delivered to the first Penning trap. There the ions are purified and again formed into a cold bunch [5]. A mass selective buffer gas cooling technique is employed that allows this trap to be operated as an isobar separator with a resolving power of up to $R \approx 10^{5}$ for ions with mass number $A=100$ [5]. The prepared ion bunch is then delivered to the second trap,

which is the high precision trap [6] used for the mass measurements of the 

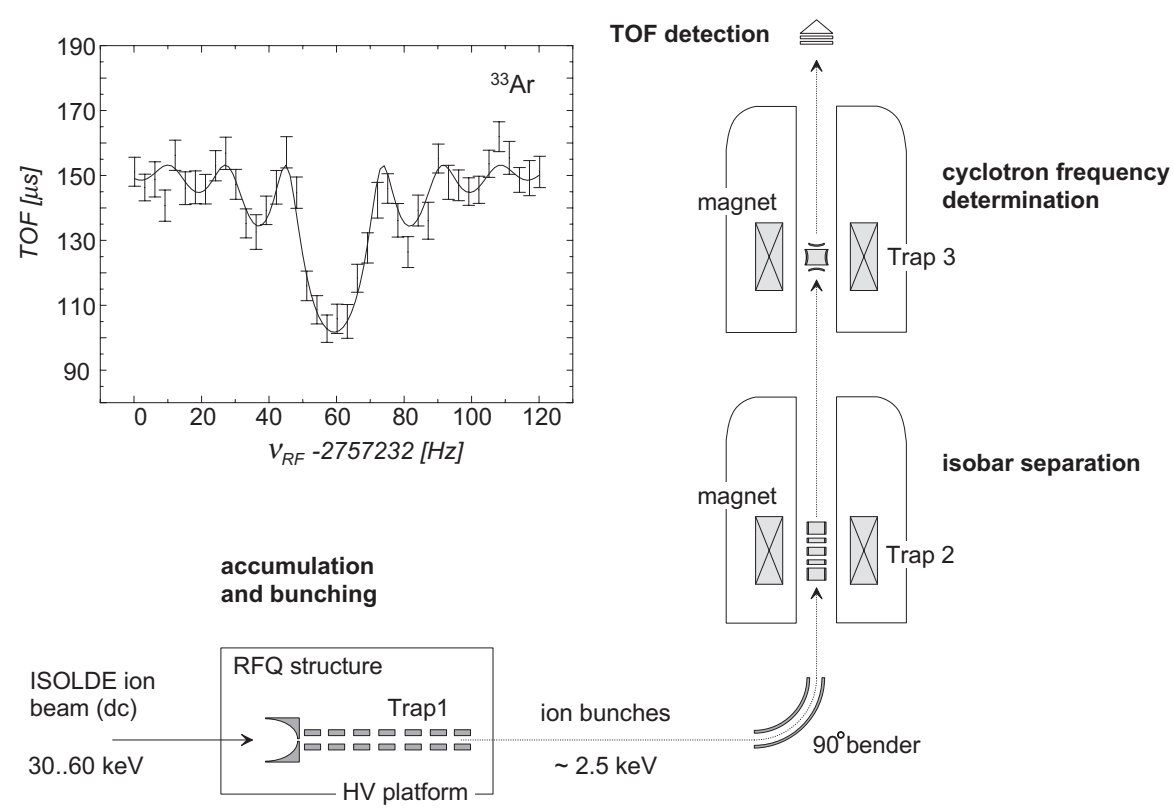

Fig. 1. Experimental setup of the ISOLTRAP mass spectrometer. The inset shows the cyclotron resonance curve for ${ }^{33} \mathrm{Ar}$.

ions. The mass measurement is carried out via a determination of the cyclotron frequency $\omega_{c}=\frac{q}{m} B$ of an ion with mass $m$ and charge $q$ in a magnetic field of known strength $B$ (determined by a reference mass). Using this technique, to date the masses of more than 100 nuclides have been determined with an accuracy of $\delta m / m \approx 1 \cdot 10^{-7}$.

\section{Mass measurements of ${ }^{33} \mathrm{Ar}$}

${ }^{33} \mathrm{Ar}\left(T_{1 / 2}=173 \mathrm{~ms}\right)$ was produced by bombarding a calcium oxide target with $1.4 \mathrm{GeV}$ protons. The radionuclides produced in the bombardment were ionized in a plasma ion source and then mass separated. The integrated yield for ${ }^{33} \mathrm{Ar}$ was in the order of a few thousand ions per proton pulse.

The ISOLDE beam gate was opened for a period of $30 \mathrm{~ms}$ at about $70 \mathrm{~ms}$ after the proton impact on the target. This time scheme maximized the yield of ${ }^{33} \mathrm{Ar}$ while minimizing the background. After accumulation the ions spent two more milliseconds for cooling in the RFQ ion beam buncher before they where ejected and transported to the first Penning trap. The total time in the first Penning trap was $73 \mathrm{~ms}$, which made it possible to reach a resolving power of $R=7000$. This was sufficient to separate ${ }^{33} \mathrm{Ar}$ from the isobars ${ }^{33} \mathrm{Cl}$ and ${ }^{33} \mathrm{~S}$ delivered by ISOLDE. The time needed to perform the actual frequency measurement in the precision trap was $70 \mathrm{~ms}$ per frequency step. The inset in Fig. 1 shows a cyclotron frequency curve for ${ }^{33} \mathrm{Ar}$ with a fit of the theoretical line shape. At the achieved resolving power of $R=130000,2000$ 
detected ions were enough to get a statistical uncertainty of $\delta m / m=9 \cdot 10^{-8}$. If the conservative estimate for the systematic error of $\delta m / m=1 \cdot 10^{-7}[7]$ is added quadratically, the mass of ${ }^{33} \mathrm{Ar}$ is then determined with a precision of $\delta m / m=1.3 \cdot 10^{-7}$, which corresponds to $4.2 \mathrm{keV}$. This reduces the error of the previous measurement [8] by a factor of 7 . In addition, the more long lived isotopes ${ }^{34,42,43} \mathrm{Ar}$ have been measured with an accuracy of $3.5 \mathrm{keV}, 5.8 \mathrm{keV}$ and $5.3 \mathrm{keV}$, respectively.

The mass value of ${ }^{33} \mathrm{Ar}$ appears to deviate from the value given by the isobaric multiplett mass equation (IMME). This would indicate that the IMME needs a cubic term for the $A=33, T=3 / 2$ quartet. This question will be the subject of a forthcoming publication.

\section{Summary and Outlook}

The installation of the new beam condition stage at the front end of the ISOLTRAP spectrometer allowed to extend the measurements to ISOLDE beams with less than a few thousand ions per second. Furthermore, due to the very fast cooling and bunching scheme, it is now possible to measure nuclides with half-lives well below one second.

In addition to the measurement of ${ }^{33} \mathrm{Ar}$, which is with $T_{1 / 2}=173 \mathrm{~ms}$ the shortest lived nuclide ever measured in a Penning trap, there was a very rich mass measurement program in 1999 on mercury [9] and xenon [10] isotopes, as well as on ${ }^{34,42,43} \mathrm{Ar}$. In the case of argon further experiments are planned to improve the accuracy and to extend the measurements to ${ }^{32} \mathrm{Ar}$.

This work was supported by the European Commision within the EUROTRAPS network under contract number ERBFMRXCT97-0144 and by NSERC of Canada.

\section{References}

[1] E. G. Adelberger et al. Phys. Rev. Lett. 83 (1999) 1299, and Phys. Rev. Lett. 83 (1999) 3101

[2] E. Kugler et al. Nucl. Instr. and Meth. B70 (1992) 41

[3] A. Kellerbauer et al. these proceedings

[4] F. Herfurth et al. in press for Nucl. Instr. and Meth. A, preprint: CERN$\mathrm{EP} / 2000-062$ 
[5] H. Raimbault-Hartmann et al. Nucl. Instr. Meth. B126 (1997) 378

[6] G. Bollen et al. Nucl. Instr. and Meth. A368 (1996) 675-697

[7] D. Beck et al. Nucl. Instr. Meth. B126 (1997) 374

[8] H. Nann et al. Phys. rev. C9 (1974) 1848

[9] S. Schwarz et al. in press for Nucl. Phys. A, preprint: CERN-EP/2001-026

[10] J. Dilling et al. these proceedings 